\title{
Case Report Systemic phaeohyphomycosis resembling primary sclerosing cholangitis caused by Exophiala dermatitidis
}

\author{
Correspondence \\ Mevlut Kurt \\ dr.mevlutkurt@gmail.com \\ Received 11 December 2008 \\ Accepted 13 May 2009
}

\author{
Erkin Oztas, ${ }^{1}$ Bulent Odemis, ${ }^{1}$ Murat Kekilli, ${ }^{1}$ Mevlut Kurt, ${ }^{1}$ \\ Bedia Mert Dinc, ${ }^{2}$ Erkan Parlak, ${ }^{1}$ Ayse Kalkanci ${ }^{3}$ and Nurgul Sasmaz ${ }^{1}$ \\ ${ }^{1}$ Department of Gastroenterology, Turkiye Yuksek Ihtisas Teaching and Research Hospital, Ankara,
Turkey \\ ${ }^{2}$ Microbiology Laboratory, Turkiye Yuksek Ihtisas Teaching and Research Hospital, Ankara, Turkey \\ ${ }^{3}$ Department of Medical Microbiology, Gazi University Faculty of Medicine, Ankara, Turkey
}

Exophiala dermatitidis, one of the saprophytic dematiaceous fungi, is a cause of local and disseminated phaeohyphomycosis. We report a case of systemic phaeohyphomycosis resembling sclerosing cholangitis caused by $E$. dermatitidis in a 24-year-old woman.

\section{Introduction}

Exophiala dermatitidis is one of the saprophytic dematiaceous fungi and causes local and disseminated phaeohyphomycosis (Nachman et al., 1996; Kenney et al., 1992). It usually occurs in persons with decreased host immunity, although cases have been reported in apparently immunocompetent patients (Sudduth et al., 1992; Bennett et al., 1973; Revankar et al., 2002; Sartoris et al., 1999; Ajello, 1974). We report a case of systemic phaeohyphomycosis caused by E. dermatitidis in a 24 -year-old woman without evidence of immunodeficiency who presented with signs resembling sclerosing cholangitis.

\section{Case report}

In May 2007, a 24-year-old woman was referred to our hospital with complaints of pruritus, right upper abdominal pain, anorexia and fatigue of 1 month duration, and jaundice and intermittent fever for several days. Five years previously, she had worked in a chicken farm for 2 years. A year previously, she had been diagnosed with tinea versicolor for a skin lesion and received treatment with oral itraconazole and a topical antifungal agent.

On physical examination, millimetric lymphadenopathies at anterior cervical and bilateral axillary regions and hepatosplenomegaly were detected. Laboratory data on admission are summarized in Table 1. Abdominal ultrasonography revealed hepatosplenomegaly, dilatation of intrahepatic bile ducts (IHBDs), diffuse thickening of the common bile duct wall, and multiple intra-abdominal (periportal, coeliac and peripancreatic) lymphadenopathies (maximum diameter of $2 \mathrm{~cm}$ ). Endoscopic retrograde

Abbreviations: CNS, central nervous system; ERC, endoscopic retrograde cholangiography; $\mathrm{HBD}$, intrahepatic bile duct. cholangiography (ERC) showed the presence of narrowed left and right main hepatic bile duct at the level of the portal hilus and bilateral IHBD dilatation. There were irregularities on the common hepatic duct and common bile duct with narrowing of the distal segments. ERC findings were suggestive of sclerosing cholangitis. After balloon dilatation of the hilar narrowed segment, stenting of the left and right main ducts was performed. Examination of a brush cytology specimen from the hilar segment revealed benign epithelial cells. After ERC, the patient had symptomatic relief. However, she insisted on discharge before an extensive work-up could be completed.

In August 2007, the woman was readmitted to our hospital with abdominal pain, intermittent fever, axillary and right anterior cervical lymphadenopathies and with a left subscapular subcutaneous nodule $2 \mathrm{~cm}$ in diameter. A thoracoabdominal CT scan revealed bilateral axillary lymphadenopathies (max. $2 \times 3 \mathrm{~cm}$ ), hepatosplenomegaly, intra-abdominal multiple enlarged lymph nodes, bilateral dilatation of the IHBD and diffuse thickening of the common bile duct wall with the previously placed intraluminal stents. Cervical lymph node excisional biopsy findings included granulomas with giant cells, yeast cells and branching hyphae (Fig. 1a). Control ERC revealed diffuse narrowing in the hilar segment with primary sclerosing cholangitis-like narrowing of the right IHBD. The stents were removed. Cytological examination of a brush specimen along with findings from a biopsy from the thickened wall of the hilar segment and common bile duct revealed the presence of pseudohyphae (Fig. 1b). Signs of acute cholangitis with precirrhotic changes and yeast cells at several portal areas were also detected on a liver biopsy (Fig. 1c). Axillary lymph node aspiration biopsy was performed and cultured on Sabouraud dextrose agar. Three days after incubation at $37^{\circ} \mathrm{C}$, light-grey colonies 
Table 1. Laboratory values

\begin{tabular}{|c|c|c|c|}
\hline & $05 / 06 / 2007$ & $30 / 11 / 2007$ & $22 / 12 / 2008$ \\
\hline White blood cell count $\left(3600-11000 \mathrm{~mm}^{-3}\right)$ & 7800 & 8200 & 8000 \\
\hline Eosinophils $(0.1-6.0 \%)$ & 15.9 & 21.8 & 1.7 \\
\hline Sedimentation rate $\left(0-20 \mathrm{~mm} \mathrm{~h}^{-1}\right)$ & 51 & 40 & 34 \\
\hline Aspartate aminotransferase $\left(0-38 \mathrm{U} \mathrm{l}^{-1}\right)$ & 122 & 58 & 16 \\
\hline Alanine aminotransferase $\left(0-41 \mathrm{U} \mathrm{l}^{-1}\right)$ & 148 & 56 & 14 \\
\hline Alkaline phosphatase $\left(0-270 \mathrm{U} \mathrm{l}^{-1}\right)$ & 1801 & 2127 & 316 \\
\hline$\gamma$-Glutamyltransferase $\left(8-61 \mathrm{U} \mathrm{l}^{-1}\right)$ & 30 & 31 & 17 \\
\hline Lactate dehydrogenase $\left(240-480 \mathrm{U} \mathrm{l}^{-1}\right)$ & 559 & 186 & 231 \\
\hline Total bilirubin $\left(0-1.1 \mathrm{mg} \mathrm{dl}^{-1}\right)$ & 4.49 & 1.41 & 0.44 \\
\hline Direct bilirubin $\left(0-0.3 \mathrm{mg} \mathrm{dl}^{-1}\right)$ & 3.72 & 0.75 & 0.17 \\
\hline $\operatorname{IgG}\left(7-16 \mathrm{~g} \mathrm{l}^{-1}\right)$ & 17.5 & & \\
\hline $\begin{array}{l}\mathrm{HbsAg} \text {, anti-HCV, anti-HIV, anti-nuclear antibody, anti-ds DNA, anti-mitochondrial } \\
\text { antibody, anti-neutrophil cytoplasmic antibody, anti-smooth muscle antibody, } \\
\text { anti-liver kidney microsome-1 antibody }\end{array}$ & Negative & & \\
\hline $\begin{array}{l}\text { Total lymphocyte count, CD4/CD8 ratio, complement 3q, complement 4, IgA and } \\
\text { IgM levels }\end{array}$ & Normal range & & \\
\hline
\end{tabular}

formed; upon further incubation, shiny black colonies appeared. Sparse, septate, and pale olivaceous hyphae were observed under a microscope and the final diagnosis of a black fungus was achieved by DNA sequencing. The D1/D2 regions of the $26 \mathrm{~S}$ rDNA were sequenced directly from PCR products using the primer pair ITS1 (5'GTCGTAACAAGGTTAACCTGCGG-3') and NL4 (5'GGTCCG TGTTTCAAGACGG-3'). DNA sequencing reaction products were analysed by using an ABI 3100 Genetic Analyzer (Applied Biosystems) and the sequence was confirmed by the NCBI gene bank as E. dermatitidis. The isolate was found to be susceptible to amphotericin B, itraconazole, voriconazole and caspofungin according to Clinical and Laboratory Standards Institute guidelines (CLSI, 2002).

The patient was initially treated with i.v. amphotericin B (a dose of $3 \mathrm{mg} \mathrm{kg}^{-1}$ per day). Three weeks later, eosinophilia regressed. After 1 month of therapy, she was switched to oral voriconazole (400 $\mathrm{mg}$ per day) and discharged from the hospital. At her next outpatient visit, there was marked improvement of her symptoms with almost normal cholestatic parameters (Table 1). The patient did not manifest any neuropsychiatric symptoms, and despite the known neurotropic nature of the fungus, extensive neurological and optic fundal examination was unremarkable. A cranial CT scan was also negative for any signs of central nervous system (CNS) infection.

\section{Discussion}

Among the obstructive causes of secondary sclerosing cholangitis, fungal infection is rarely seen. Although disseminated infections with Candida and other fungal species have increasingly been recognized, fungal involvement of the biliary tract is extremely rare. E. dermatitidis is a mould with a yeast synanamorph found in the environment. It is a rare cause of human infection that, when invasive, is nearly always fatal (Nachman et al., 1996; Kenney et al., 1992).

Previous reports have emphasized the high mortality rate associated with disseminated $E$. dermatitidis infection because of the neurotropic nature of this organism. Matsumoto et al. (1993) reviewed 37 cases of phaeohyphomycosis caused by E. dermatitidis. In 20 cases, there was evidence of systemic disease, including 12 fatal disseminated infections with involvement of the lymph nodes, CNS, endocardium, eye and lungs. Most of the patients died due to CNS involvement. According to this paper, 18 patients were immunocompetent and had no underlying diseases.

Hiruma et al. (1993) reported a case of systemic E. dermatitidis infection where the patient had symptoms of obstructive cholangitis due to an inflammatory mass in the region of the head of the pancreas and duodenum. The patient was treated by providing external biliary drainage and by the administration of antifungal agents but died 3 years after initial presentation due to CNS involvement. However, this case differs from ours by the fact that in our case the obstructive cholangitis symptoms were due to direct involvement of the biliary tree by $E$. dermatitidis. In another case report, a patient had cholestatic symptoms as well as lymphadenopathies on first presentation, and later developed biliary cirrhosis. However, the dematiaceous fungus which led to phaeohyphomycosis could not be identified (Harish et al., 2004).

The route of infection is obscure in many cases, usually with the absence of identifiable skin or mucous membrane lesions. It is possible that the source of the fungus is exogenous in nature, either inhaled into the lungs with dust after which invasion takes place, or ingested with food and thus enters the digestive tract, which is followed by 


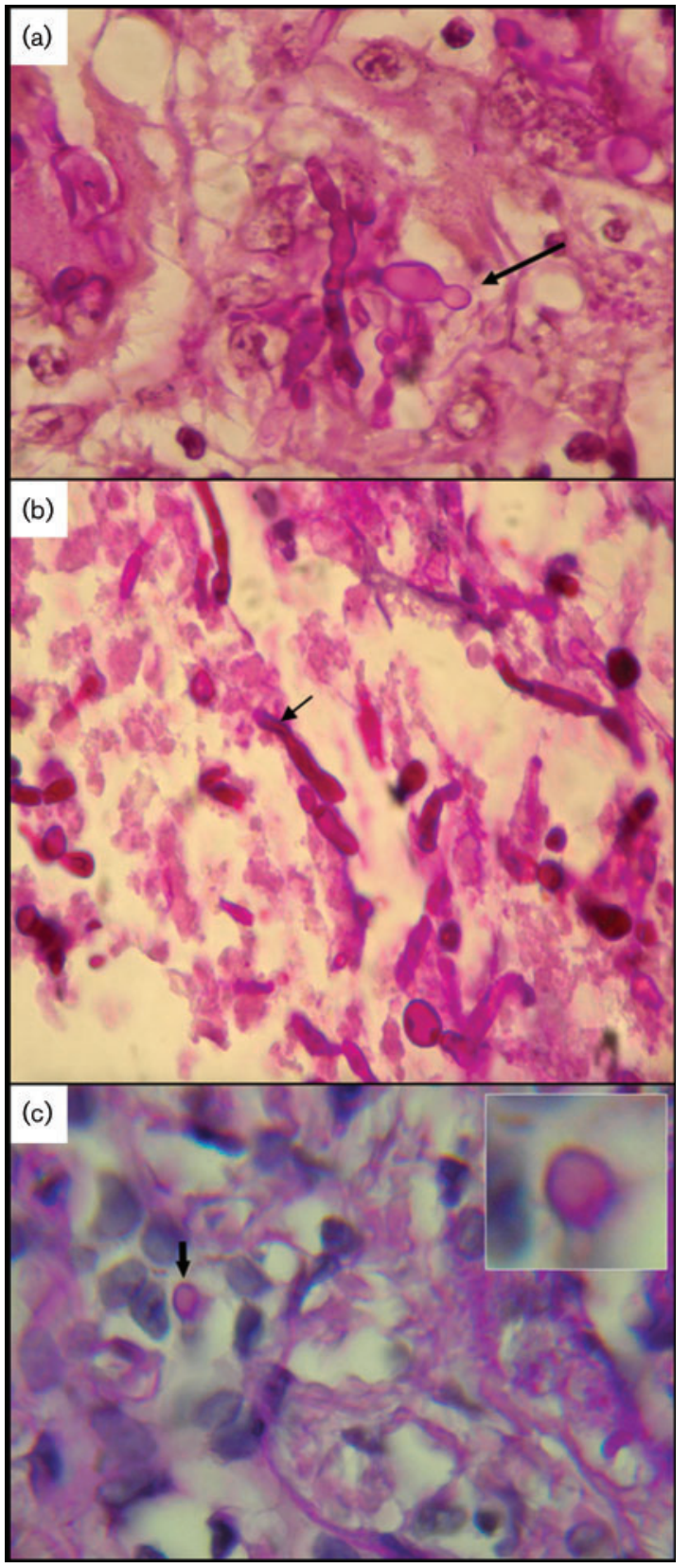

Fig. 1. (a) Yeast cells and branching hyphae in a cervical lymph node excisional biopsy (periodic acid-Schiff stain $\times 200$ ). (b) Fungal elements (yeast cells and irregular branching hyphae) in a biopsy from the thickened wall of the hilar segment (periodic acidSchiff stain $\times 100$ ). (c) Yeast cell in a liver biopsy (haematoxylin and eosin stain).

retrograde passage into the biliary tract and then the bloodstream. Our patient did not give a history of close contact with an individual with cystic fibrosis. Moreover, although there were no skin lesions at presentation, our patient had a previous history of tinea versicolor diagnosed and treated by a primary care general practitioner. This history led us to speculate that the initial skin lesion was perhaps due to $E$. dermatitidis, and that the ensuing systemic infection may have been due to traumatic inoculation. Though E. dermatitidis is an agent known to be common in European steam baths (Matos et al., 2002), the patient had no history of being in such an environment. She had previously worked in a chicken farm, and it is possible that she was exposed to $E$. dermatitidis during that period. Of course, it is not possible to establish this for certain.

Current therapeutic options for these devastating and frequently fatal infections are limited, and fungal infections of this kind are still notoriously resistant to treatment. Furthermore, the optimum treatment period is still unknown. According to previously reported data, when the initial small, localized, cutaneous and subcutaneous lesions are amenable to surgical excision, such a procedure would be successful in managing the infection. However, in the case of disseminated infection, chemotherapy with amphotericin B, flucytosine, itraconazole or combination therapy should be attempted (Matsumoto et al., 1993; Sharkey et al., 1990; McGinnis \& Pasarell, 1998b). Nevertherless, disseminated infection is extremely difficult to treat and many of the patients in such cases died due to CNS involvement despite therapy. Therefore, several authors have iterated the need for new effective antifungal agents. Voriconazole, posaconazole and ravuconazole are new potent antifungal agents against dematiaceous fungi and hold promise for improving the therapy of those infections (McGinnis \& Pasarell, 1998a; Hay, 1999; Perez, 1999; Cuenca-Estrella et al., 1999).

To the best of our knowledge, this is the first report on phaeohyphomycosis due to E. dermatitidis with a clinical presentation resembling primary sclerosing cholangitis. Sclerosing cholangitis due to direct involvement of the biliary tree deserves to be documented as a complication of systemic E. dermatitidis infection.

\section{References}

Ajello, L. (1974). Phaeohyphomycosis: Definition and Etiology. Scientific publication 304. Washington, DC: Pan American Health Organization.

Bennett, J. E., Bonner, H., Jennings, A. E. \& Lopez, R. I. (1973). Chronic meningitis caused by Cladosporium Trichoides. Am J Clin Pathol 59, 398-407.

CLSI (2002). Reference Method for Broth Dilution Antifungal Susceptibility Testing of Filamentous Fungi; Approved Standard M38-A. Wayne, PA: Clinical and Laboratory Standards Institute.

Cuenca-Estrella, M., Ruiz-Díez, B., Martínez-Suárez, J. V., Monzón, A. \& Rodriguez-Tudela, J. L. (1999). Comparative in vitro activity of voriconazole and six other antifungal agents against clinical isolates of Scedosporium prolificans and Scedosporium apiospermum. J Antimicrob Chemother 43, 149-151.

Harish, K., Harikumar, R., Aravindan, K. P. \& Varghese, T. (2004). Hepatobiliary phaeohyphomycosis with secondary biliary cirrhosis. Trop Gastroenterol 25, 172-173.

Hay, R. J. (1999). Therapeutic potential of terbinafine in subcutaneous and systemic mycoses. Br J Dermatol 141, 36-40. 
Hiruma, M., Kawada, A., Ohata, H., Ohnishi, Y., Takahashi, H., Yamazaki, M., Ishibashi, A., Hatsuse, K., Kakihara, M. \& Yoshida, M. (1993). Systemic phaeohyphomycosis caused by Exophiala dermatitidis. Mycoses 36, 1-7.

Kenney, R. T., Kwon-Chung, K. J., Waytes, A. T., Melnic, D. A., Pass, H. I., Merino, M. J. \& Gallin, J. I. (1992). Successful treatment of systemic Exophiala dermatitidis infection in a patient with chronic granulomatous disease. Clin Infect Dis 14, 235-242.

Matos, T., de Hoog, G. S., de Boer, A. G., de Crom, I. \& Haase, G. (2002). High prevalence of the neurotrope Exophiala dermatitidis and related oligotrophic black yeasts in sauna facilities. Mycoses 45, 373-377.

Matsumoto, T., Matsuda, T., McGinnis, M. R. \& Ajello, L. (1993). Clinical and mycological spectra of Wangiella dermatitidis infections. Mycoses 36, 145-155.

McGinnis, M. R. \& Pasarell, L. (1998a). In vitro evaluation of terbinafine and itraconazole against dematiceous fungi. Med Mycol 36, 243-246.

McGinnis, M. R. \& Pasarell, L. (1998b). In vitro testing of susceptibilities of filamentous ascomycetes to voriconazole, itraconazole and amphotericin B, with consideration of phylogenetic implications. J Clin Microbiol 36, 2353-2355.
Nachman, S., Alpan, O., Malowitz, R. \& Spitzer, E. D. (1996). Catheter-associated fungemia due to Wangiella (Exophiala) dermatitidis. J Clin Microbiol 34, 1011-1013.

Perez, A. (1999). Terbinafine: broad new spectrum of indications in several subcutaneous and systemic and parasitic diseases. Mycoses 42, 111-114.

Revankar, S. G., Patterson, J. E., Sutton, D. A., Pullen, R. \& Rinaldi, M. G. (2002). Disseminated phaeohyphomycosis: review of emerging mycosis. Clin Infect Dis 34, 467-476.

Sartoris, K. E., Baillie, G. M., Tiernan, R. \& Rajagopalan, P. R. (1999). Phaeohyphomycosis from Exphiala jeanselmei with concomitant Nocardia asteroides infection in a renal transplant recipient: case report and review of the literature. Pharmacotherapy 19, 995-1001.

Sharkey, P. K., Graybill, J. R., Rinaldi, M. G., Stevens, D. A., Tucker, R. M., Peterie, J. D., Hoeprich, P. D., Greer, D. L., Frenkel, L. \& other authors (1990). Itraconazole treatment of phaeohyphomycosis. J Am Acad Dermatol 23, 577-586.

Sudduth, E. J., Crumbley, A. J., III \& Farrar, W. E. (1992). Phaeohyphomycosis due to Exophiala species: clinical spectrum of disease in humans. Clin Infect Dis 15, 639-644. 